\title{
Modeling the Distance-Dependent Blurring in Transmission Imaging in the Ordered-Subset Transmission (OSTR) Algorithm by Using an Unmatched Projector/Backprojector Pair
}

\author{
B. Feng, Member, IEEE, M. A. King, Senior Member, IEEE, H. C. Gifford, Member, IEEE, P. H. \\ Pretorius, Associate Member, IEEE, G. L. Zeng, Senior Member, IEEE, and J. A. Fessler, Senior \\ Member, IEEE
}

\begin{abstract}
In SPECT, accurate emission reconstruction requires attenuation compensation with high-quality attenuation maps. Resolution loss in transmission maps could cause blurring and artifacts in emission reconstruction. For a transmission system employing parallel-hole collimators and a sheet source, distance-dependent blurring is caused by the non-ideal source and camera collimations, and the finite intrinsic resolution of the detector. These can be approximately modeled by an incrementalblurring model. To compensate for this blurring in iterative transmission reconstruction, we incorporated the incremental blurring model in the forward projector of the OSTR algorithm but did not include the blur in the backprojector. To evaluate our approach, we simulated transmission projections of the MCAT phantom using a ray-tracing projector, in which the rays coming out from a source point form a narrow cone. The geometric blurring due to the non-ideal source and camera collimations was simulated by multiplying the counts along each cone-beam ray with a weight calculated from the overall geometric response function (assumed a two-dimensional Gaussian function), and then summing the weighted counts into projections. The resulting projections were convolved with the intrinsic response (another two-dimensional Gaussian) to simulate the total system blurring of transmission imaging. Poisson noise was then added to the projection data. We also acquired two sets of transmission measurements of an air-filled Data Spectrum Deluxe SPECT phantom on a Prism 2000 scanning-line-source transmission system. We reconstructed the simulations using the OSTR algorithm, with and without modeling of the incremental blur in the projector. The scaling parameter of the penalty prior was optimized in each case by minimizing the root-mean-square error
\end{abstract}

Manuscript received November 5, 2005. This work was supported by grant HL50349 from the National Heart, Lung, and Blood Institute. The contents are solely the responsibility of the authors and do not necessarily represent the official views of the National Institutes of Health.

B. Feng, M. A. King, H. C. Gifford, and P. H. Pretorius are with the Department of Radiology, University of Massachusetts Medical School, Worcester, MA 01655. (telephone: 508-856-8795, e-mail: Bing.Feng@umassmed.edu).

J. A. Fessler is with the Department of Electrical Engineering and Computer Science, University of Michigan, Ann Arbor, MI 48109. (e-mail: fessler@umich.edu).

G. L. Zeng is with the Department of Radiology, University of Utah, Salt Lake City, UT 84108. (e-mail: larry@ucair.med.utah.edu).
(RMSE). Reconstructions showed that modeling the incremental blur improved the resolution of the attenuation map and quantitative accuracy.

\section{INTRODUCTION}

$\mathrm{T}^{\mathrm{N}}$ SPECT, accurate emission reconstruction requires performing attenuation compensation with high quality attenuation maps [1-3]. Attenuation maps are usually obtained through transmission imaging systems which could employ various transmission sources and imaging geometries. The finite resolution of a transmission system causes discrepancies in the origins and detected locations of transmission photons, which results in the blurring of attenuation maps. The resolution of transmission systems has been studied mostly for optimization of system designs [4, 5]. However, there have been some investigations into compensating for the blurring in transmission reconstruction [6-8]. It is challenging to model both the distance-dependent blurring and Poisson statistical nature of the transmission imaging in reconstruction algorithms, since the measurement model is non-linear in terms of the linear attenuation coefficient.

In [7], we proposed and evaluated an approximate method which models the average blurring of an object in the orderedsubset transmission (OSTR) algorithm [9]. In this manuscript, we proposed an alternative method which more accurately models the blurring of a transmission imaging system than the method of modeling the average blurring. Under the framework of using an unmatched projector/backprojector pair [10], we investigated a method of modeling the incremental blurring through the Gaussian diffusion technique [11] in the OSTR algorithm. The incremental blurring was modeled only when calculating the average transmission projections (in the projector), not in the backprojector. Though our method could be extended to other geometries, throughout this work we focus on the transmission system employing SPECT camera with the sheet source and parallel-hole collimation. In such a system, the recorded counts are the superposition of the contributions from many positions in the photon source. An ideal approach would treat this like an "overlapping beams" model [12]. The overlapping beams method works well when there are just a few photon sources. Herein there is a 
continuum of sources so the overlapping beams approach becomes computationally inconvenient. Instead, we approximate the effects of the superposition by modifying the forward projector. This is an approximation because of the nonlinearity of Beer's law, and such models cannot account for the "exponential edge-gradient effect" [13].

\section{METHODS}

\section{A. Modeling of the Distance-Dependent Blurring of Parallel-Hole Collimated Transmission System by Using an Incremental Slice-by-Slice Blurring Model}

For a transmission system that uses sheet source and parallel-hole camera collimator (as shown in Fig. 1), the sheet source is usually collimated by parallel-hole source collimator. In Figure 1, the attenuating object is divided into slices which are of equal thickness $(\Delta \mathrm{d})$ and parallel to camera. An arbitrary oblique ray is investigated to evaluate the blurring due to the non-ideal source and camera collimations. We assumed that the distance between the sheet source and source collimator is negligible, and so is the distance between camera and camera collimator. $\mathrm{D}$ is the distance from the source to camera. $\mathrm{D}_{1}$, and $\mathrm{D}_{2}$ are the distances from the first and second slice to camera, respectively. $\mathrm{D}_{\text {last }}$ is the distance from the last slice to camera. $\Delta \mathrm{d}$ is the slice thickness, one pixel is our current choice. $t_{1}, t_{2}$, and $t_{3}$ are the in-plane offsets from distances $D$, $\mathrm{D}_{1}, \mathrm{D}_{2}$ on the oblique ray to the intersection, respectively.

We assumed that the geometric response function for the source collimator is a two-dimensional (2D) Gaussian with sigma of $\sigma_{s}$, and the geometric response function for the camera collimator is another (2D) Gaussian with sigma of $\sigma_{c}$. Considering a photon traveling along the arbitrary oblique path as the solid line in Figure 1, the chance of the photon passing through both source and camera collimators is the multiplication of the chances passing through each alone. It is straightforward to show that the overall geometric response function is a $2 \mathrm{D}$ Gaussian with sigma of

$$
\sigma_{g}=1 / \sqrt{1 / \sigma_{s}^{2}+1 / \sigma_{c}^{2}}
$$

which depends on the distance $\mathrm{D}$ between the source and camera, because $\sigma_{s}$ and $\sigma_{c}$ depend on D. Since a 2D Gaussian can be separated into two 1D Gaussians along each dimension, we focus the investigations on just the in-plane dimension (as in Fig. 1) and will extend our reasoning to the other dimension without further explanations.

We could model the blurring due to the non-ideal source and camera collimations as overlapping cone-beams which are irradiated from each source bin with the probability determined by the overall geometric response function. In iterative reconstruction this overlapping cone-beam approach is too computational expensive. Thus we need find an approximate approach which traces photon paths only parallel to collimation. As inspired by the incremental blurring model in emission imaging $[11,14]$, we proposed to model the geometric blur in transmission imaging with an incremental attenuating and blurring model. In each angular view, the object is divided into equally-thick slices parallel to the camera surface. This is achieved through the 2D Gaussian rotator [15] which rotates the image grids with the camera head. For photons along the oblique ray shown in Figure 1, the chance of passing through the first slice (imagine there is only one slice and there is no scattering) and arriving at the detector is proportional to $e^{-t^{2} /\left(2 \sigma_{g}^{2}\right)}$ which is equal to $e^{-t_{1}{ }^{2} /\left(2 \sigma_{1}^{2}\right)}$ or $e^{-t_{2}{ }^{2} /\left(2 \sigma_{2}^{2}\right)}$, where $\sigma_{1}=\left(D_{1} / D\right) \sigma_{g}$ and $\sigma_{2}=\left(D_{2} / D\right) \sigma_{g}$ (see Fig. 1). This indicates that the geometric blur is proportional to the distance from the camera, as is well known. The incremental blur in the first slice could be approximated by a Gaussian with sigma of $\Delta \sigma_{1}=\sqrt{\sigma_{1}^{2}-\sigma_{2}^{2}}$. Similarly we move on to the next slice, and so on. In tracing the rays through the object the transmission photons go through a series of incremental attenuation and blurring steps.

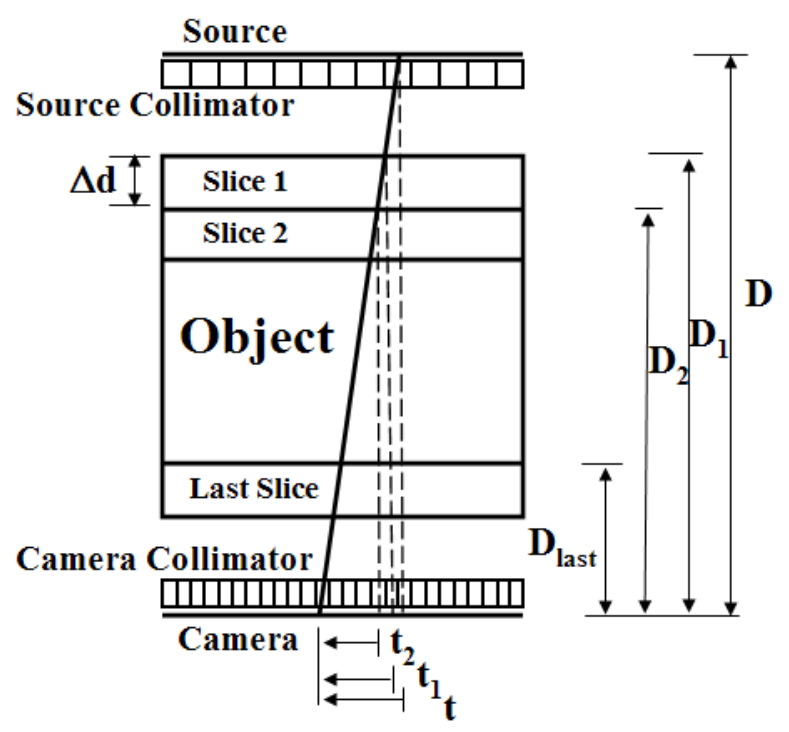

Fig. 1. Drawing of a transmission system that uses a sheet source collimated by a parallel-hole collimator and a parallel-hole collimator on the opposed camera head. The attenuating object is divided into slices which are of equal thickness $(\Delta \mathrm{d}$, one pixel herein) and parallel to the camera. An arbitrary oblique ray is investigated to evaluate the blur due to the non-ideal source and camera collimations.

At the last slice blur due to the intrinsic resolution (with sigma of $\sigma_{i}$ ) should be taken into account. The resultant sigma for the last slice is $\Delta \sigma_{\text {last }}=\sqrt{\sigma_{\text {last }}^{2}+\sigma_{i}^{2}}$, where $\sigma_{\text {last }}=\left(D_{\text {last }} / D\right) \sigma_{g}$. In brief with our approach, there are two parameters describing the blurring of a transmission system: the slope $\sigma_{g} / D$, and the intercept $\sigma_{i}$.

The ordered-subset transmission algorithm (OSTR) [9] is derived from the Poisson statistical model, and can be easily incorporated with smoothness priors. In the OSTR algorithm, the average counts for each detector bin are calculated from the known flood and the estimated attenuation map. In calculation of the average transmission projections, we use a ray-driven projector that models the slice-by slice incremental attenuation 
and blurring of photons. In the backprojector, the blurring is neglected.

Smoothness priors are normally used in the OSTR algorithm to penalize the roughness of the attenuation maps reconstructed, and to generate a physical solution to the illposed inverse problem built in the reconstruction. The smoothness prior we used is an edge-preserving prior with the potential function of the form

$$
\psi(t)=\beta \delta^{2}\left(\sqrt{1+t^{2} / \delta^{2}}-1\right),
$$

where $\beta$ is a scaling parameter which will be determined through the MCAT simulations, and $\sigma$ is the threshold that is empirically set as $1 / 20$ the attenuation coefficient of lungs at $140 \mathrm{keV}$ (about $0.0025 \mathrm{~cm}^{-1}$ ) [16].

\section{B. The MCAT Phantom Simulations and Optimization of Scaling Parameter of the Smoothness Prior}

We simulated a 180-degree transmission acquisition of the MCAT phantom using overlapping cone-beams, modeling the geometric response, intrinsic resolution, and attenuation (Fig. 2). A uniform flood was assumed of 36 counts per pixel (with $0.416 \mathrm{~cm}$ pixel size), which was about the count-level for a standard 20-minute scan on Prism 2000 system. The distance between the transmission source and the detector was assumed $\mathrm{D}=60 \mathrm{~cm}$. Photons come out from each source bin as from a point-source, and follow cone-beam paths arriving at the detector. The effect of collimations is reflected in the overall geometric response function $\left(\sigma_{g} / D=0.02\right)$. Counts along each cone-beam ray were initially equal to the flood strength multiplied by the geometric response function, then attenuated by the object, and finally summed into each detector bin to form projection data. The linear integral of the attenuation coefficient was calculated by a ray-driven projector. The projection data were convolved with the intrinsic response $\left(\sigma_{i}=0.12 \mathrm{~cm}\right)$ to simulate the intrinsic blur of the detector.

Poisson noise was then added to the projection data, which consisted of 60 views of $128 \times 128$ pixels. The noisy projection data were reconstructed with 50 iterations of the OSTR algorithm, with and without modeling the incremental blurring. For each case the scaling parameter of the smoothness prior was optimized in terms of the root-mean-square error (RMSE). The RMSE was defined in a $3 \mathrm{D}$ region-of-interest (ROI) including the entire thorax as

$$
R M S E=\sqrt{\frac{\sum_{i \in R O I}\left(\mu_{i}-\mu_{i}^{\text {true }}\right)^{2}}{N}},
$$

where $\mathrm{N}$ is the number of voxels inside the ROI, $\mu_{i}$ and $\mu_{i}^{\text {true }}$ are the reconstructed and true values of the linear attenuation coefficient at voxel $i$, respectively. With and without modeling the incremental blurring, an optimal $\beta$ was found in each case using the minimum RMSE as the criterion. The attenuation maps reconstructed with the optimal $\beta$ stands for the most accurate results which can be achieved in each case. The minimum RMSE with and without modeling distance-dependent resolution were compared with each other, and also with the minimum RMSE obtained with modeling the average blurring [7]. On the attenuation maps reconstructed with the optimal $\beta$, the RMSE on each slice was also calculated by

$$
R M S E_{Z}=\sqrt{\frac{\sum_{i \in R_{Z}}\left(\mu_{i}-\mu_{i}^{\text {true }}\right)^{2}}{N_{Z}}},
$$

where $\mathrm{ROI}_{Z}$ is the region of interest on the slice $\mathrm{Z}(\mathrm{Z}=20,21$, $22, \ldots, 100), N_{z}$ is the number of voxels inside $\mathrm{ROI}_{z}$. The $\mathrm{RMSE}_{Z}$ was compared on every slice for the three cases described earlier.
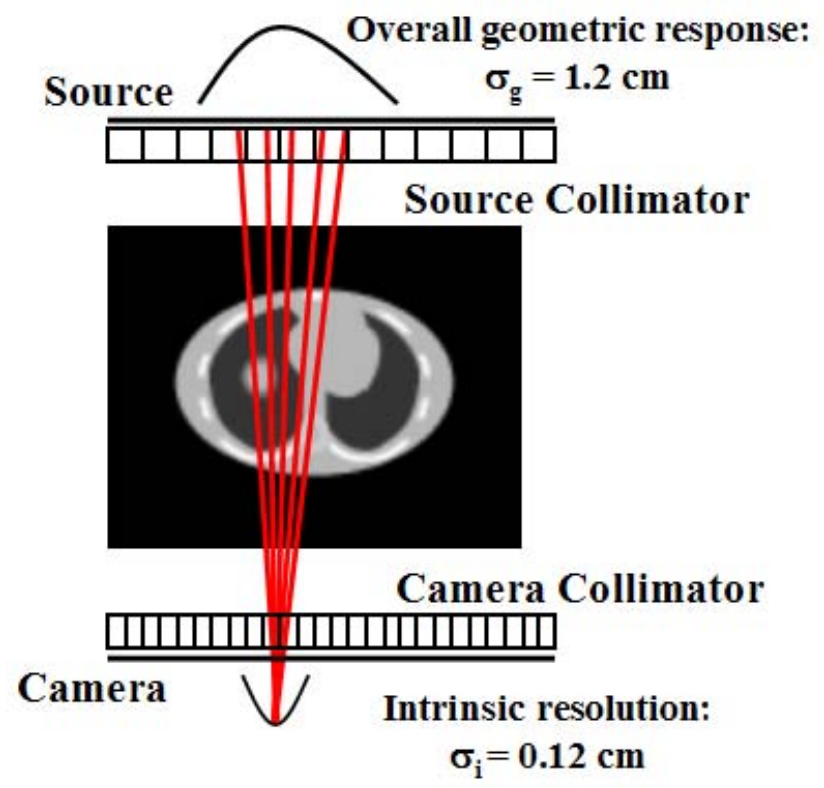

Fig. 2. The transmission system with sheet source and parallel-hole camera collimation as used in simulation. The cone-beam rays in the simulation are plotted in red solid lines. Counts were summed into each detector bin through the cone-beam rays, modeling geometric response, attenuation, and the intrinsic resolution of the camera.

\section{Experimental Study with the Air-Filled Deluxe SPECT Phantom}

Two 30 minute transmission acquisitions of the air-filled Data Spectrum Deluxe SPECT phantom were performed on a PRISM 2000 SPECT system [17] which uses a scanning linesource for transmission imaging. The two acquisitions differed from each other by the radius of rotation (ROR) employed: $18.5 \mathrm{~cm}$ in one case, and $30 \mathrm{~cm}$ the other. Each acquisition was reconstructed with 50 iterations of the OSTR algorithm with and without modeling the incremental blurring. Since we are interested herein in the in-plane resolution only, we treated the scanning line-source system as a sheet-source system, assuming the same in-plane and axial resolution. The parameters describing the incremental blurring were chosen as $\sigma_{g} / D=0.02$, and $\sigma_{i}=0.12 \mathrm{~cm}$. The scaling parameter $\beta$ was chosen such that the best reconstruction of the air-filled deluxe SPECT phantom was obtained visually. 


\section{RESULTS}

\section{A. The MCAT Phantom Simulations and Optimization of the} Scaling Parameter of the Smoothness Prior

The simulated transmission projections were reconstructed with a range of $\beta$ from 0 to 10000 with a step-size of 500, with and without modeling the incremental blurring. The RMSE in the 3D ROI is plotted versus the scaling parameter $\beta$ in Figure 3 . In the case of not modeling the blurring, the minimum RMSE was obtained at $\beta=4000$, with a value of $0.027 \mathrm{~cm}^{-1}$. In case of modeling the incremental blurring, the minimum RMSE was $0.020 \mathrm{~cm}^{-1}$, with $\beta=5000$. Modeling of the incremental blurring helped increase the accuracy of the reconstruction. However, compared with reconstruction of the same data with modeling the average blurring [7], in which the minimum RMSE was $0.017 \mathrm{~cm}^{-1}$, modeling of the incremental blurring was sub-optimal.

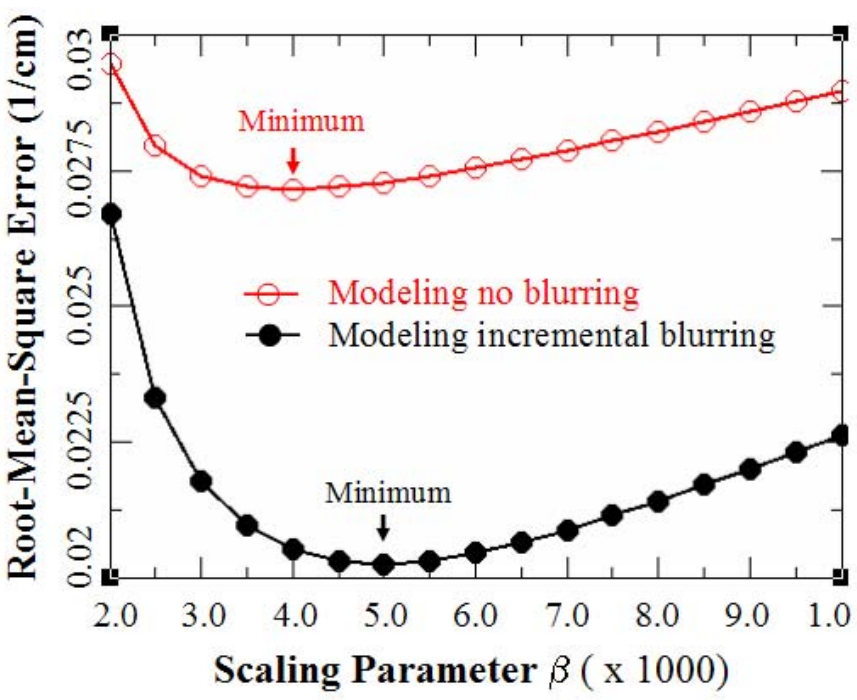

Fig. 3. Optimization of the scaling parameter of the smoothness prior in reconstruction of the MCAT phantom simulation, using 50 iterations of the OSTR, with and without modeling the incremental blurring. The minimum RMSE that can be achieved is smaller with modeling the incremental blurring $\left(0.020 \mathrm{~cm}^{-1}\right)$ than without modeling the incremental blurring $\left(0.027 \mathrm{~cm}^{-1}\right)$.

The attenuation maps reconstructed with the optimal scaling parameter $\beta$ are shown in Figure 4. The map reconstructed with modeling the incremental blurring is smoother yet has a better outline of the body and lungs than the attenuation map reconstructed without modeling the blurring. The root-meansquare error on each slice $\left(\mathrm{RMSE}_{\mathrm{Z}}\right.$ in Eq. 4) is plotted versus the slice $\mathrm{Z}$ in Figure 5, for modeling the incremental blurring and without blurring. Also is plotted the $\mathrm{RMSE}_{\mathrm{Z}}$ for modeling the average blurring. Among the three cases, modeling of the average blurring gives the least

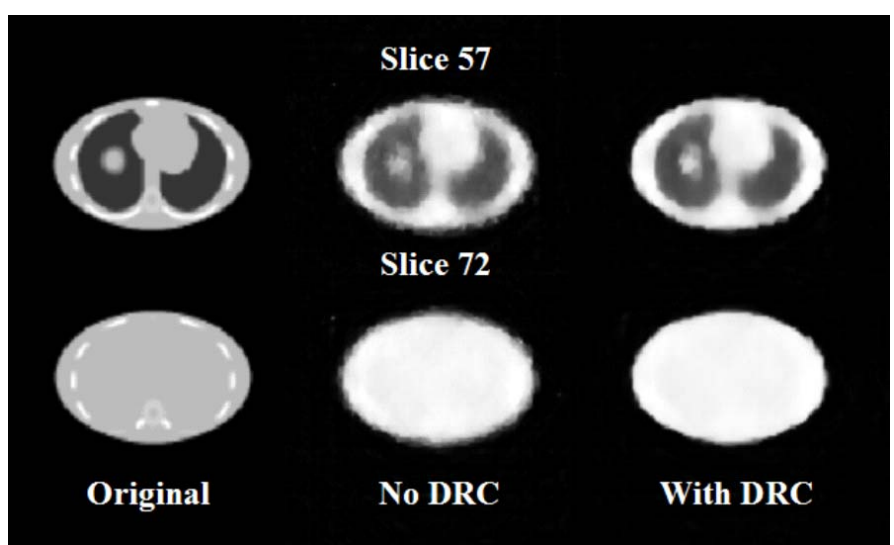

Fig. 4. Reconstruction of the projection data simulated for the transmission system shown in Fig. 2, with the optimal scaling parameter $\beta$ of the smoothness prior determined as that which minimized the root-mean-square error (RMSE) in each case (Fig. 3). (Left) Two slices of the original MCAT phantom. (Middle) The same slices from 50 iterations of the OSTR without modeling the incremental blurring $(\beta=4000)$. (Right) The same slices from 50 iterations of the OSTR with modeling the incremental blurring $(\beta=5000)$.

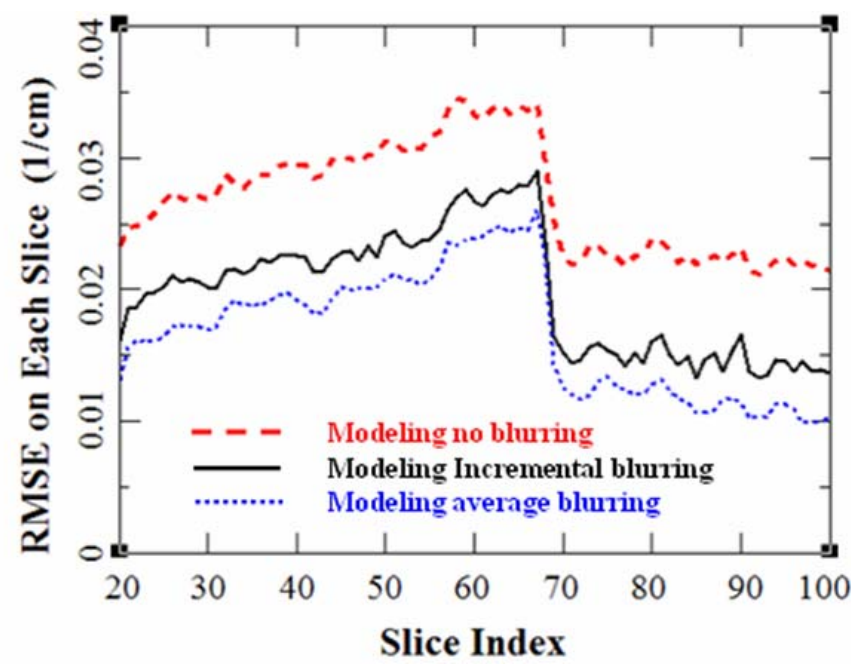

Fig. 5. The root-mean-square error on each slice $\left(\mathrm{RMSE}_{z}\right.$ in Eq. 4) is plotted versus the slice index $\mathrm{Z}$ for modeling no blurring (dashed line), modeling the average blurring (solid line), and modeling the incremental blurring (dotted line).

$\mathrm{RMSE}_{\mathrm{Z}}$ on every slice. Yet modeling of the incremental blurring shows smaller $\mathrm{RMSE}_{Z}$ on every slice than modeling of no blurring. In each case reduced $\mathrm{RMSE}_{Z}$ was observed in the slice range containing the uniform soft tissue. An abrupt dropping of the $\mathrm{RMSE}_{Z}$ happens in the transition range where the lungs become too small to contribute much to $\mathrm{RMSE}_{\mathrm{Z}}$, and finally vanish. Before the abrupt decrease, the $\mathrm{RMSE}_{\mathrm{Z}}$ has a tendency of increasing with the slice index for each case since the lungs become smaller and smaller. This causes an increasing partial volume effect which reduces the overall accuracy. 


\section{B. Experimental Study with the Air-Filled Deluxe SPECT Phantom}

The results of reconstructing the measurements of the airfilled Deluxe SPECT phantom are shown in Figure 6. Better resolution is seen on the reconstructions with modeling the incremental blurring, as shown in the right row in Figure 6.

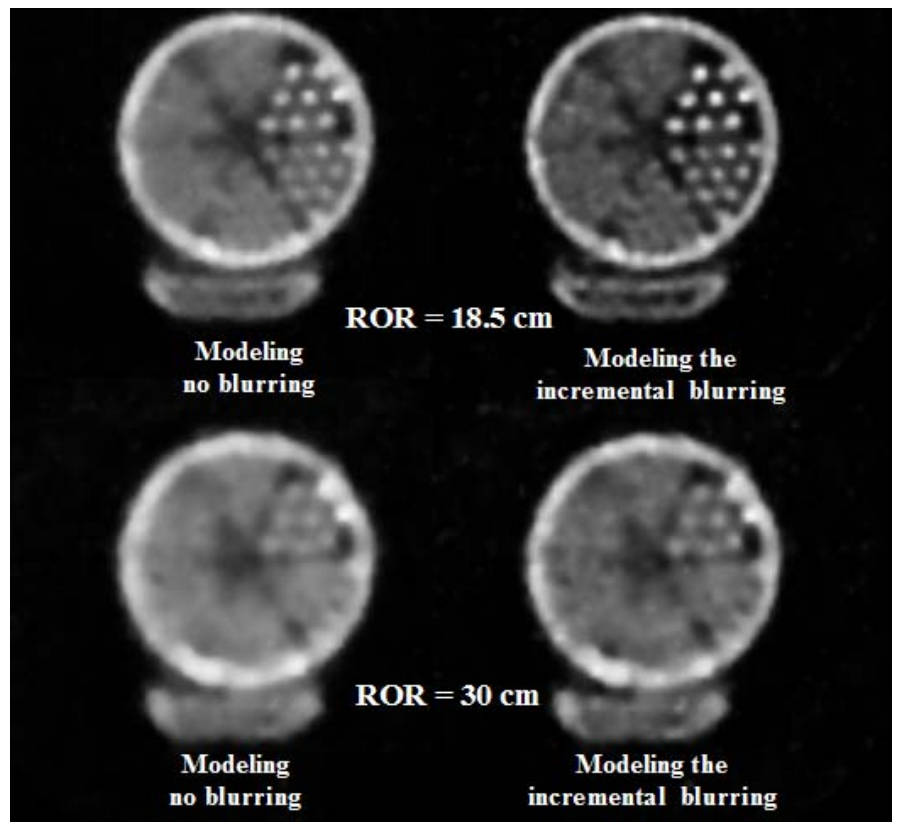

Fig. 6. Reconstruction of experimental data for the air-filled Data Spectrum Deluxe SPECT phantom. (Upper left) From acquisition using a $18.5 \mathrm{~cm}$ ROR, without modeling the blurring. (Upper right) From acquisition using a $18.5 \mathrm{~cm}$ ROR, with modeling the incremental blurring. (Lower left) From acquisition using a $30 \mathrm{~cm}$ ROR, without modeling the blurring. (Upper right) From acquisition using a $30 \mathrm{~cm}$ ROR, with modeling the incremental blurring.

\section{DISCUSSION}

The method of modeling the incremental blurring in the projector only shows improved reconstruction accuracy over modeling no blurring. Yet it is shown less accurate than modeling the average blurring while using matched projector/backprojector in the OSTR algorithm. A possible remedy could be modeling the incremental blurring in projector and modeling the average blurring in the backprojector.

\section{CONCLUSION}

Modeling of the distance-dependent blurring using the incremental blurring model in the OSTR algorithm increases the resolution and quantitative accuracy of the transmission reconstruction for a parallel-hole collimated SPECT system. With the improved attenuation map, more accurate attenuation correction might be achieved.

\section{REFERENCES}

[1] Tsui BMW, X. D. Zhao, D. S. Lalush, E. C. Frey, R. E. Johnston, L. S. Bujenovic, and W. H. McCartney, "Pitfalls of attenuation compensation and their remedies in cardiac SPECT", Society of Nuclear Medicine 41st Annual Meeting, Orlando, Florida, June 5-8, 1994.

[2] M. A. King, B. M. W. Tsui, and T.-S. Pan, "Attenuation compensation for cardiac single-photon emission computed tomography imaging: Part 1. Impact of attenuation and methods of estimating attenuation maps," $J$. Nucl. Card., vol. 2, pp. 513-524, 1995.

[3] A. Celler, K. L. Dixon, Z. Chang, S. Blinder, J. Powe, and R. Harrop, "Problems created in attenuation-corrected SPECT images by artifacts in attenuation maps: A simulation Study," J. Nucl. Med., vol. 46, pp. 335343, 2005.

[4] B. J. Kemp, F. S. Prato, and R. L. Nicholson, "The geometric modulation transfer function of a transmission imaging system that uses a SPECT scintillation camera and parallel hole collimation," Med. Phys., vol. 26, pp. 733-741, 1995.

[5] Z. J. Cao and B. M. W. Tsui, "Performance characteristics of transmission imaging using a uniform sheet source with parallel-hole collimation," Med. Phys., vol. 19, pp. 1205-1212, 1992.

[6] J. M. Ollinger, "Maximum-likelihood reconstruction of transmission images in emission computed tomography via the EM algorithm," IEEE. Tr. Med. Im., vol. 21, pp. 89-101, 1994.

[7] B. Feng, J. A. Fessler, and M. A. King, "Incorporation of the Detector Resolution Compensation (DRC) in the Ordered-Subset Transmission (OSTR) Algorithm for Transmission Imaging in SPECT," submitted to Tr. Med. Im.

[8] J. E. Bowsher, M. P. Tornai, J. Peter, D. E. González Trotter, A. Krol, D. R. Gilland, and R. J. Jaszczak, "Modeling the axial extension of a transmission line source within iterative reconstruction via multiple transmission sources," IEEE Tr. Med. Im., vol. 21, pp. 200-215, 2002.

[9] H. Erdoğan and J. A. Fessler, "Ordered Subsets Algorithms for Transmission Tomography," Phys. Med. Biol., vol. 44, pp. 2835-51, 1999.

[10] G. L. Zeng, and G. T. Gullberg, "Unmatched Projector/Backprojector Pairs in an Iterative Reconstruction Algorithm," IEEE Trans Med Imag, vol 19, pp. 548-555, 2000.

[11] A. W. McCarthy and M. I. Miller, "Maximum Likelihood SPECT in Clinical Computation Times Using Mesh-Connected Parallel Computers," IEEE. Tr. Med. Im., vol. 10, pp. 426-436, 1991.

[12] D. F. Yu, J. A. Fessler, and E. P. Ficaro, "Maximum Likelihood Transmission Image Reconstruction for Overlapping Transmission Beams," IEEE Tr. Med. Im., vol. 19, pp. 1094-1105, 2000.

[13] P. M. Joseph and R. D. Spital, "The exponential edge-gradient effect in x-ray computed tomography," Phys. Med. Biol., vol. 26, pp. 473-487, 1981.

[14] C. Bai, G. L. Zeng, G. T. Gullberg, F. DiFilippo and S. Miller, "Slab-byslab blurring model for geometric point response correction and attenuation correction using iterative reconstruction alogorithms," IEEE. Trans. Nucl. Sci., vol. 45, pp. 2168-2173, 1998

[15] J. W. Wallis, and T. R. Miller, "An optimal rotator for iterative reconstruction," IEEE Trans Med Imag, vol. 16, pp. 118-123, 1997.

[16] B. Feng, J. A. Fessler, P. H. Pretorius, R. D. Beach, G. L. Zeng, and M. A. King, "Evaluation of the Ordered-Subset Transmission (OSTR) Algorithm for Transmission Imaging on SPECT Systems Using Axially Overlapping Cone-Beams," submitted to IEEE. Trans. Nucl. Sci.

[17] P. Tan, D. L. Bailey, S. R. Meikle, S. Eberl, R. R. Fulton, and B. F. Hutton, "A scanning line source for simultaneous emission and transmission measurements in SPECT," J. Nucl. Med., vol. 34, pp. 1752$1760,1993$. 